Pacific Journal of Mathematics

THE BEHAVIOR OF SOLUTIONS OF A LINEAR
DIFFERENTIAL EQUATION OF SECOND ORDF 


\title{
THE BEHAVIOR OF SOLUTIONS OF A LINEAR DIFFERENTIAL EQUATION OF SECOND ORDER
}

\author{
RICHARD A. MOORE
}

Introduction. This paper is a study of the oscillation and boundedness of solutions of the self-adjoint differential equation

$$
\left(r(x) y^{\circ}\right)^{\prime}+p(x) y=0
$$

on the infinite half-axis $l, a \leq x<+\infty$. We shall assume throughout that $r(x)$ and $p(x)$ are real, continuous functions and that $r(x)$ is positive on $I$. A nonnull solution of equation (1) is said to be oscillatory if it has an infinity of zeros on $l$.

It will be noted that the results given here are of the "integral test" variety. Although the problem goes back at least to Kneser [5,6]; probably the first "integral" condition for oscillation is due to Fite [1]. His criterion is that all solutions of the even order equation

$$
y^{(2 n)}+p(x) y=0
$$

oscillate provided $p(x)>0$ and

$$
\int_{a}^{\infty} p(x) d x=+\infty
$$

A similar result for the case $n=1$ is due to Wintner [14] in which there is no restriction on the sign of $p(x)$. Simultaneously Leighton [8] noted the analogous result for equation ( 1 ) (see Theorem 1 in this paper).

Hille [2] studied the nonoscillation of solutions of (1) for the case $r(x) \equiv 1$ and $p(x)$ nonnegative and established the roles of the functions

$$
\int_{a}^{x} \xi p(\xi) d \xi \text { and } x \int_{x}^{\infty} p(\xi) d \xi
$$

Received August 2, 1953. A substantial part of the results of this paper were obtained while the author was employed on contract AF18 (600)-419 with the Office of Scientific Research of the Air Research and Development Command of the Air Force.

The author is indebted to Professor Walter Leighton for many helpful suggestions in the preparation of this paper.

Pacific J. Math. 5 (1955), 125 - 145 
(see Theorems 3 and 4, and the remarks preceding Theorem 6 in this paper).

The chief purpose of this paper is to extend the results of Hille and Leighton.

1. Oscillation theorems. We shall recall first the theorem due to Leighton.

THEOREM 1. If both

$$
\int_{a}^{\infty} \frac{d x}{r(x)}=+\infty \text { and } \int_{a}^{\infty} p(x) d x=+\infty
$$

hold, then the solutions of equation (1) are oscillatory.

It has been pointed out [10] that if the conditions of the theorem fail to hold, an oscillation-preserving substitution

$$
y=u(x) z, u(x)>0,
$$

will frequently transform equation ( 1 ) into a form in which the conditions are applicable. Indeed, under the assumption that $\left(r(x) u^{\prime}(x)\right)^{\prime}$ be continuous, $z$ satisfies the self-adjoint equation

$$
\left(r(x) u^{2}(x) z^{\prime}\right)^{\prime}+\left[u(x)\left(r(x) u^{\prime}(x)\right)^{\prime}+u^{2}(x) p(x)\right] z=0 .
$$

We make the observation that solutions oscillate if and only if there is an admissible $u(x)$ such that

$$
\int_{a}^{\infty} \frac{d x}{r(x) u^{2}(x)}=+\infty, \int_{a}^{\infty}\left[u(x)\left(r(x) u^{\prime}(x)\right)^{\prime}+u^{2}(x) p(x)\right] d x=+\infty
$$

This follows from Theorem 1 and the fact that a function $u_{1}(x)$ can be exhibited ( see, for example, $[10]$ ) which satisfies the identity

$$
\frac{1}{r(x) u_{1}^{2}(x)} \equiv u_{1}(x)\left(r(x) u_{1}^{\prime}(x)\right)^{\prime}+u_{1}^{2}(x) p(x) .
$$

In this case, a particular solution of $(1.1)$ is clearly

$$
\sin \left(\int_{a}^{x} \frac{d \xi}{r(\xi) u_{1}^{2}(\xi)}\right)
$$

which oscillates only if $\int_{a}^{\infty}\left(r(\xi) u_{1}^{2}(\xi)\right)^{-1} d \xi=+\infty$. 
We proceed with the proof of the following result.

THEOREM 2. Solutions of equation (1) are oscillatory provided conditions (A) or (B) are satisfied:

(A)

$$
\begin{aligned}
& \int_{a}^{\infty} \frac{d x}{r(x)}=+\infty, \text { and, for some } n<1 \\
& \int_{a}^{\infty} p(x) g^{n}(x) d x=+\infty \text {, where } g(x)=1+\int_{a}^{x} \frac{d \xi}{r(\xi)} . \\
& \int_{a}^{\infty} \frac{d x}{r(x)}<+\infty, \text { and, for some } m>1 \\
& \int_{a}^{\infty} p(x) h^{m}(x) d x=+\infty, \text { where } h(x)=\int_{x}^{\infty} \frac{d \xi}{r(\xi)} .
\end{aligned}
$$

To prove the theorem, note that if (A) holds, equation (1) may be transformed by the substitution

$$
y=g^{n / 2}(x) z
$$

The function $z(x)$ satisfies the equation

$$
\left(r_{1}(x) z^{\prime}\right)^{\prime}+p_{1}(x) z=0
$$

where

$$
r_{1}(x)=r(x) g^{n}(x), p_{1}(x)=\frac{(n / 2)(n / 2-1)}{r(x) g^{2-n}(x)}+p(x) g^{n}(x)
$$

Theorem 1 is now applicable, for

$$
\int_{a}^{\infty} \frac{d x}{r_{1}(x)}=\left.\lim _{x \rightarrow \infty} \frac{g^{1-n}(x)}{1-n}\right|_{a} ^{x}=+\infty
$$

and

$$
\int_{a}^{\infty} p_{1}(x) d x=\left.\lim _{x \rightarrow \infty} \frac{(n / 2)(n / 2-1)}{n-1} g^{n-1}(\xi)\right|_{a} ^{x}+\int_{a}^{\infty} p(x) g^{n}(x) d x=+\infty
$$

therefore, solutions of (1.2) (and, thus, those of (1)) oscillate. 
If conditions (B) hold, the substitution $y=h^{n / 2}(x) z$ leads to the following equation for $z$

$$
\left(r_{2}(x) z^{\prime}\right)^{\prime}+p_{2}(x) z=0
$$

where

$$
r_{2}(x)=r(x) h^{m}(x), p_{2}(x)=\frac{(m / 2)(m / 2-1)}{r(x) h^{2-m}(x)}+p(x) h^{m}(x) .
$$

An application of Theorem 1 completes the proof.

If the conditions of Theorem 2 are not satisfied, we shall see later that some results can be obtained by studying the functions

$$
\int_{a}^{x} p(\xi) g(\xi) d \xi \quad \int_{a}^{x} p(\xi) h(\xi) d \xi
$$

The following result, due to Hille [2, p. 238], is given for completeness. It has been reformulated here so that it applies to equation ( 1 ).

THEOREM 3. If

$$
\int_{a}^{\infty}|p(x)| g(x) d x<+\infty
$$

the general solution of equation (1) is

$$
c_{1}\left(1+e_{1}(x)\right)+c_{2} g(x)\left(1+e_{2}(x)\right),
$$

where $c_{1}$ and $c_{2}$ are arbitrary and $\lim _{x \rightarrow \infty} e_{i}(x)=0(i=1,2)$.

An obvious modification of Hille's proof of Theorem 3 yields the following result.

THE OREM 4. If

$$
\int_{a}^{\infty}|p(x)| h(x) d x<+\infty
$$

the general solution of equation (1) is

$$
c_{1}\left(1+e_{1}(x)\right)+c_{2} h(x)\left(1+e_{2}(x)\right),
$$


where $\lim _{x \rightarrow \infty} e_{i}(x)=0 \quad(i=1,2)$.

This theorem trivially implies that all solutions of equation (1) are both nonoscillatory and bounded.

The following is a similar nonoscillation test, applicable to a broader class of equations than those covered by the two preceding theorems.

THEOREM 5. Solutions of equation (1) are nonoscillatory if either of the following conditions is satisfied:

(A) There exists a finite number $A$ such that, for $x \geq b \geq a$,

$$
0<A-\int_{b}^{x} p(\xi) g(\xi) d \xi<1
$$

(B) There exists a finite number $B$ such that for $x \geq b \geq a$,

$$
0<B+\int_{b}^{x} p(\xi) h(\xi) d \xi<1
$$

Suppose that Condition (A) is satisfied and consider the differential equation

$$
\left(r_{1}(x) y^{\prime}\right)^{\prime}+p(x) y=0,
$$

where

$$
r_{1}(x)=r(x)\left[A-\int_{b}^{x} p(\xi) g(\xi) d \xi\right]
$$

We have, according to Condition (A), that $0<r_{1}(x)<r(x)$ for $b \leq x<+\infty$. According to the Sturm comparison theorem, solutions of equation (1) are nonoscilatory if those of equation (1.3) are. We see, however, that $g(x)$ is a particular solution of equation (1.3). Since $g(x)$ is nonoscillatory, all solutions of (1.3) are, and the proof of the result is completed.

To prove the theorem in the case where Condition (B) holds, note that the equation

$$
\left(r(x)\left[B+\int_{b}^{x} p(\xi) h(\xi) d \xi\right] y^{\prime}\right)^{\prime}+p(x) y=0
$$

has the particular solution $h(x)$. The proof is completed by the use of the same 
argument as that employed in case (A).

We include an example which shows that the theorem is not valid under either of the less restrictive assumptions:

$$
\max _{x \geq a} \int_{a}^{x} p(\xi) g(\xi) d \xi-\min _{x \geq a} \int_{a}^{x} p(\xi) g(\xi) d \xi<M
$$

$$
\max _{x \geq a} \int_{a}^{x} p(\xi) h(\xi) d \xi-\min _{x \geq a} \int_{a}^{x} p(\xi) h(\xi) d \xi<M,
$$

where $M$ is any finite number.

EXAMPLE 1. Consider the equation

$$
\left(x^{2} y^{\circ}\right)^{\prime}-A_{1} \cos (2 \log x) y=0
$$

on the interval $[1,+\infty)$, where $A_{1}$ is a parameter. A computation shows that

$$
\max _{x \geq 1} \int_{1}^{x} p h d \xi-\min _{x \geq 1} \int_{1}^{x} p h d \xi=A_{1} \text {. }
$$

On the other hand the substitution $y=x^{-1 / 2} z$ and the subsequent transformation $t=\log x$ yield a function $w(t)=z(x(t))$, which satisfies the Mathieu equation

$$
\frac{d^{2} w}{d t^{2}}+\left(-\frac{1}{4}-A_{1} \cos 2 t\right) w=0
$$

We assert that solutions of equation $(1.4)^{\prime}$ (and thus those of equation (1.4)) are oscillatory if $\left|A_{1}\right|$ is larger than a critical constant $A_{2}$ where the approximate value of $A_{2}$ is 1.5 . To show this we recall several properties of the solutions of the general Mathieu equation

$$
y^{\prime \prime}+(c-q \cos 2 x) y=0,
$$

where $c$ and $q$ are real parameters. It is known (for example, Ince [3]) that for number pairs $(c, q)$, which satisfy the equation

$$
c=f_{0}(q),
$$

equation (1.4)" has a periodic nonoscillating solution, which we denote by $u_{q}(x)$. The function $f_{0}(q)$ has the properties that it is continuous and single 
valued for $-\propto<q<+\infty$. In addition $f_{0}(0)=0, f_{0}(q)$ is an even function of $q$, and $f_{0}(q)$ is monotone decreasing for $q>0$. It follows that, if $\left|q_{1}\right|>q_{0}$, solutions of

$$
y^{\prime \prime}+\left(f_{0}\left(q_{0}\right)-q_{1} \cos 2 x\right) y=0
$$

are oscillatory. To see this, we transform equation $(1.4)^{\prime \prime \prime}$ by the substitution $y=u_{q_{1}}(x) z$, into

$$
\left(u_{q_{1}}^{2}(x) z^{\prime}\right)^{\prime}+u_{q_{1}}^{2}(x)\left[f_{0}\left(q_{0}\right)-f_{0}\left(q_{1}\right)\right] z=0 .
$$

Since $f_{0}\left(a_{0}\right)-f_{0}\left(a_{1}\right)$ is a positive constant, the application of Theorem 1 shows that solutions of (1.4)"'" are oscillatory.

(Note that, if $\left|q_{1}\right| \leq q_{0}$ in equation (1.4) "', the above technique establishes the fact that solutions of equation (1.4) " vestigation of Example 1 has thus led to a complete description of the oscillating and nonoscillating cases for the Mathieu equation.)

We complete the proof of the assertion by noting that

$$
-\frac{1}{4}=f(q)
$$

has the solutions $q= \pm A_{2}[4]$.

Hille has proved the following result $[2, \mathrm{p} .246]$ for the case where $r(x)=1$ and $p(x) \geq 0$ in equation (1).

If

$$
x \int_{x}^{\infty} p(\xi) d \xi \leq \frac{1}{4}
$$

solutions of equation ( 1 ) are nonoscillatory. If, on the other hand,

$$
x \int_{x}^{\infty} p(\xi) d \xi \geq c>\frac{1}{4}
$$

solutions of (1) oscillate.

We obtain a generalization of this result, valid for any admissible $r(x)$ and free of the assumption that $p(x)$ be nonnegative. If $\int_{x}^{\infty} p(\xi) d \xi$ exists, we define 


$$
G(x)=g(x) \int_{x}^{\infty} p(\xi) d \xi,
$$

and if $\int_{x}^{\infty}(1 / r(\xi)) d \xi$ exists, we define

$$
H(x)=h(x) \int_{a}^{x} p(\xi) d \xi .
$$

THEOREM 6. If there exists a real number $k$ such that either of the following inequalities holds for $a \leq b \leq x<+\infty$.

$$
\begin{aligned}
& -k-\sqrt{k} \leq G(x) \leq-k+\sqrt{k} \leq \frac{1}{4}, \\
& -k-\sqrt{k} \leq H(x) \leq-k+\sqrt{k} \leq \frac{1}{4},
\end{aligned}
$$

then solutions of equation (1) are nonoscillatory.

If $k$ is such that the first inequalities hold, we prove the result by forming the function

$$
u(x)=g^{k}(x) \exp \left\{\int_{a}^{x} \frac{1}{r(\xi)} \int_{\xi}^{\infty} p(s) d s d \xi\right\}
$$

A computation shows that $u(x)$ is a solution of the differential equation

$$
\left(r(x) u^{\prime}\right)^{\prime}+p_{1}(x) u=0,
$$

where

$$
p_{1}(x)=p(x)-\left\{\frac{k(k-1)+2 k G(x)+G^{2}(x)}{r(x) g^{2}(x)}\right\} .
$$

The function $p_{1}(x)$ is continuous; moreover, by hypothesis, for $x \geq b \geq a$, $G(x)$ assumes only values on the closed interval determined by the two roots of the quadratic equation

$$
t^{2}+2 k t+k(k-1)=0
$$

It follows that

$$
k(k-1)+2 k G(x)+G^{2}(x) \leq 0 ;
$$


hence, for $x \geq b, p_{1}(x) \geq p(x)$. Since solutions of equation (1.5) are nonoscillatory, an application of the Sturm comparison theorem completes the result.

In case the second inequality holds, we form, for the appropriate $k$, the function

$$
w(x)=h^{k}(x) \exp \left\{-\int_{a}^{x} \frac{1}{r(\xi)} \int_{a}^{\xi} p(t) d t d \xi\right\}
$$

This function is a solution of the differential equation

$$
\left(r(x) w^{\prime}\right)^{\prime}+p_{2}(x) w=0,
$$

where

$$
p_{2}(x)=p(x)-\left\{\frac{k(k-1)+2 k H(x)+H^{2}(x)}{r(x) h^{2}(x)}\right\} .
$$

The remaining details of the proof are identical with those of the first case.

Corresponding to the second half of Hille's theorem we have the following, THEOREM 7. If either

$$
\frac{1}{4}<c \leq G(x) \leq d<+\infty
$$

or

$$
\frac{1}{4}<c \leq H(x)
$$

holds, then solutions of equation (1) are oscillatory. If $p(x) \geq 0, G(x)$ need not be bounded from above.

Note first that if $G(x) \geq c>0$, then

$$
\int_{a}^{\infty} \frac{d x}{r(x)}=+\infty
$$

We consider the following identities:

$$
\int_{a}^{x} p(\xi) g(\xi) d \xi=\int_{a}^{x} \frac{G(\xi)}{r(\xi) g(\xi)} d \xi-G(x)+\int_{a}^{\infty} p(x) d x
$$




$$
\int_{a}^{x} p(\xi) h(\xi) d \xi=\int_{a}^{x} \frac{H(\xi)}{r(\xi) h(\xi)} d \xi+H(x)
$$

It follows from the conditions of the theorem that either

$$
\int_{a}^{x} p(\xi) g(\xi) d \xi \geq c \int_{a}^{x} \frac{d \xi}{r(\xi) g(\xi)}-d+O(1)
$$

or

$$
\int_{a}^{x} p(\xi) h(\xi) d \xi \geq c \int_{a}^{x} \frac{d \xi}{r(\xi) h(\xi)}
$$

Equation (1) for the substitutions, $y=g^{1 / 2}(x) z$, or $y=h^{1 / 2}(x) z$, respective$l y$, is transformed into

$$
\left(r(x) g(x) z^{\prime}\right)^{\prime}+\left[-\frac{1}{4 r(x) g(x)}+p(x) g(x)\right] z=0
$$

or

$$
\left(r(x) h(x) z^{\prime}\right)^{\prime}+\left[-\frac{1}{4 r(x) h(x)}+p(x) h(x)\right] z=0,
$$

respectively. We can now apply Theorem 1 . We have for equation (1.6)

$$
\int_{a}^{\infty} \frac{d x}{r(x) g(x)}=+\infty
$$

and

$$
\begin{array}{r}
\lim _{x \rightarrow \infty} \int_{a}^{x}\left[\frac{-1}{4 r(\xi) g(\xi)}+p(\xi) g(\xi)\right] d \xi \geq\left(c-\frac{1}{4}\right) \lim _{x \rightarrow \infty} \int_{a}^{x} \frac{d \xi}{r(\xi) g(\xi)} \\
-d+O(1)=+\infty
\end{array}
$$

In similar fashion Theorem 1 is applicable to equation $(1.6)^{\prime}$ to show that, in either case, solutions of ( 1 ) oscillate.

If $p(x) \geq 0$ and $\int_{a}^{\infty}(1 / r(x)) d x=+\infty$ an obvious modification of Hille's proof $[2$, p. 242] yields the result. 
Corollary 1. Solutions of equation (1) are nonoscillatory if both

$$
\int_{a}^{\infty} \frac{d x}{r(x)}<+\infty \text { and } \limsup _{x \rightarrow \infty}\left|\int_{a}^{x} p(\xi) d \xi\right|<+\infty
$$

The proof follows immediately from the fact that $\lim _{x \rightarrow \infty} H(x)=0$.

This result is a refinement of a theorem due to Leighton [9, p. 657] which states that the result holds if $r(x) p(x)$ is a positive, monotone function and both $p(x)$ and $1 / r(x)$ have convergent infinite integrals.

That the constant $1 / 4$ is sharp is shown by the example of the Euler equation

$$
y^{\prime \prime}+\frac{a^{2}}{x^{2}} y=0
$$

The following example shows that the condition

$$
-\infty<L_{0} \leq G(x) \leq L_{1} \leq \frac{1}{4}
$$

is not sufficient for nonoscillation.

EXAMPLE 2. Consider the equation

$$
\left(x^{7 / 8} y^{\prime}\right)^{\prime}+\left(-\frac{63}{256}+A \cos (2 \log x)\right) x^{-9 / 8} y=0
$$

on the interval $[1, \infty)$. The substitutions $y=x^{1 / 16} z$ and $t=\log x$ show that equation (1.7) is only the transformed Mathieu equation

$$
\frac{d^{2} z}{d t^{2}}+\left(-\frac{1}{4}+A \cos 2 t\right) z=0 .
$$

It was established in Example 1 that this equation (and hence equation (1.7)) has oscillating solutions if, for example $A=2>A_{2}$. A computation of $G(x)$ for equation (1.7) shows that

$$
G(x)=\frac{-63}{4}-8 A\left(\frac{256}{257}\right)\left(\frac{\sin (2 \log x)}{2}-\frac{\cos (2 \log x)}{32}\right)+o(1) .
$$

For this $A$ we have the desired result; that is, 


$$
-\infty<L_{0} \leq G(x) \leq L_{1} \leq \frac{1}{4}
$$

If in equation (1) $r(x)=1$, we make the substitution $y=x^{m} z, m<1 / 2$; thus, $z$ satisfies the equation

$$
\left(x^{2 m} z^{\prime}\right)^{\prime}+\left(m(m-1) x^{2 m-2}+x^{2 m} p(x)\right) z=0 .
$$

Computing $G(x)$ for this equation, we find

$$
G(x)=\frac{m(m-1)}{(2 m-1)^{2}}+\frac{x^{1-2 m}}{1-2 m} \int_{x}^{\infty} \xi^{2 m} p(\xi) d \xi+o(1) .
$$

We define

$$
G_{m}(x)=x^{1-2 m} \int_{x}^{\infty} p(\xi) d \xi,
$$

and obtain the following extension of Hille's theorem.

COROLLARY 2. If in equation (1) $r(x)=1$, and for $x \geq x_{0} \geq a$ and $m<1 / 2$ we have

$$
0<G_{m}(x)<2 m,
$$

then solutions of equation (1) are nonoscillatory.

Since

$$
\frac{m(m-1)}{(2 m-1)^{2}}=\frac{-m^{2}}{(1-2 m)^{2}}-\frac{m}{1-2 m}=-k-\sqrt{k}
$$

and

$$
\frac{m(m-1)}{(2 m-1)^{2}}+\frac{2 m}{1-2 m}=\frac{-m^{2}}{(1-2 m)^{2}}+\frac{m}{1-2 m}=-k+\sqrt{k},
$$

under the assumptions on $G_{m}(x)$, the function $G(x)$ for equation (1.8) satisfies

$$
-k-\sqrt{k} \leq G(x) \leq-k+\sqrt{k} \text {. }
$$

The corollary now follows from Theorem 6. 
$G_{1 / 2}(x)$ is defined only if $\lim _{x \rightarrow \infty} \int_{a}^{x} \xi p(\xi) d \xi$ exists and is finite, and thus, according to Theorem 5 , the solutions are nonoscillatory. In any case, this result is a natural link between Hille's theorem and Theorem 5 .

We shall denote by $N_{y}\left(x_{1}, x_{2}\right)$ the number of zeros of a solution $y(x)$ of equation ( 1 ) on the interval $\left[x_{1}, x_{2}\right]$. According to the Sturm separation theorem, for any other solution $u(x)$ of (1)

$$
N_{u}\left(x_{1}, x_{2}\right)=N_{y}\left(x_{1}, x_{2}\right)+O(1) ;
$$

therefore, there will be no ambiguity in calling $N\left(x_{1}, x_{2}\right)$ the number of zeros on $\left[x_{1}, x_{2}\right]$ of any solution of equation (1).

In order to place estimates on $N\left(x_{1}, x_{2}\right)$, we note that equation (1) is equivalent to the system of equations

$$
y^{\prime}=\frac{a(x)}{r(x)} v, \quad v^{\prime}=\frac{-p(x)}{a(x)} y-\frac{a^{\prime}(x)}{a(x)} v,
$$

where $a(x)>0$ and has a continuous derivative. In terms of polar coordinates $R$ and $\Theta$ in the $y v$-plane, we have

$$
\begin{aligned}
& -\frac{d \Theta}{d x}=\frac{a(x)}{r(x)} \sin ^{2} \Theta+\frac{p(x)}{a(x)} \cos ^{2} \Theta+\frac{a^{\prime}(x)}{a(x)} \sin \Theta \cos \Theta \\
& \frac{d(\log R)}{d x}=\left[\frac{a(x)}{r(x)}-\frac{p(x)}{a(x)}\right] \cos \Theta \sin \Theta-\frac{a^{\prime}(x)}{a(x)} \sin ^{2} \Theta .
\end{aligned}
$$

Every solution $y(x)$ of equation (1) can be uniquely represented by

$$
y(x)=R(x) \cos \Theta(x)
$$

where $[R(x), \Theta(x)]$ is a solution of the system (1.9) (1.10). These equations can be exploited for oscillation and boundedness theorems, typical of which is the following.

THEOREM 8. For any constant $A>0$,

$$
\begin{aligned}
& N\left(x_{1}, x_{2}\right) \leq \frac{1}{\pi} \int_{x_{1}}^{x_{2}} \max \left[\frac{A}{r(x)}, \frac{p(x)}{A}\right] d x+O(1) \\
& N\left(x_{1}, x_{2}\right) \geq \frac{1}{\pi} \int_{x_{1}}^{x_{2}} \min \left[\frac{A}{r(x)}, \frac{p(x)}{A}\right] d x+O(1) .
\end{aligned}
$$


To see this, we set $a(x)=A$; thus, equation (1.9) becomes

$$
-\frac{d \Theta}{d x}=\frac{A}{r(x)} \sin ^{2} \Theta+\frac{p(x)}{A} \cos ^{2} \Theta .
$$

It is clear that

$$
N\left(x_{1}, x_{2}\right)=\frac{1}{\pi} \int_{x_{1}}^{x_{2}}\left(-\frac{d \Theta}{d x}\right) d x+O(1)
$$

The result now follows from the obvious inequalities

$$
\min \left[\frac{A}{r(x)^{\prime}} \frac{p(x)}{A}\right] \leq \frac{A}{r(x)} \sin ^{2} \Theta+\frac{p(x)}{A} \cos ^{2} \Theta \leq \max \left[\frac{A}{r(x)}, \frac{p(x)}{A}\right] .
$$

THEOREM 9. If $r(x) p(x) \leq M^{2}$ for $a \leq x<+\infty$ then

$$
\limsup _{x \rightarrow \infty} \int_{a}^{x} p(\xi) d \xi=+\infty
$$

is a sufficient, and

$$
\int_{a}^{\infty} \frac{d x}{r(x)}=+\infty
$$

a necessary condition that solutions of equation (1) oscillate. $^{1}$

To prove the result note that for $A=M$

$$
\min \left[\frac{A}{r(x)}, \frac{p(x)}{A}\right]=\frac{p(x)}{M} \text { and } \max \left[\frac{A}{r(x)}, \frac{p(x)}{A}\right]=\frac{M}{r(x)} .
$$

From these relations and Theorem 8 , we have that

$$
\frac{1}{\pi M} \int_{a}^{x} p(\xi) d \xi+O(1) \leq N(a, x) \leq \frac{M}{\pi} \int_{a}^{x} \frac{d \xi}{r(\xi)}+O(1)
$$

The remaining details of the proof are now obvious.

We remark that

${ }^{1}$ This theorem was suggested by an unpublished theorem due to Leighton and Martin in which the condition $|r(x) p(x)|<M$ replaces the analogous condition here. 


$$
\max \left[\frac{A}{r(x)}, \frac{p(x)}{A}\right]-\min \left[\frac{A}{r(x)}, \frac{p(x)}{A}\right]=\left|\frac{A}{r(x)}-\frac{p(x)}{A}\right|
$$

hence, if for some constant, $A$,

$$
\int_{a}^{\infty}\left|\frac{A}{r(x)}-\frac{p(x)}{A}\right| d x<\infty,
$$

then

$$
N(a, x)=\frac{A}{\pi} \int_{a}^{x} \frac{d \xi}{r(\xi)}+O(1) .
$$

Equation (1.9) can be written as follows

$$
-\frac{d \Theta}{d x}=\frac{1}{2}\left[\frac{A}{r(x)}+\frac{p(x)}{A}\right]-\frac{1}{2}\left[\frac{A}{r(x)}-\frac{p(x)}{A}\right] \cos 2 \Theta .
$$

THEOREM 10. If for some $A$

$$
\lim _{x \rightarrow \infty}\left[\frac{A}{r(x)}-\frac{p(x)}{A}\right]\left[\frac{A}{r(x)}+\frac{p(x)}{A}\right]^{-1}=0,
$$

then

$$
N(a, x)=\frac{1+e(x)}{\pi} \int_{a}^{x} \frac{1}{2}\left[\frac{A}{r(\xi)}+\frac{p(\xi)}{A}\right] d \xi,
$$

where $\lim _{x \rightarrow \infty} e(x)=0$.

The proof follows immediately from equation (1.11).

2. Theorems on boundednes. We derive here a number of results by suitable choices of $a(x)$ in equation (1.10).

THEOREм 11. For every $A>0$ we have

$$
\exp \left\{-\frac{1}{2} \int_{a}^{x}\left|\frac{A}{r}-\frac{p}{a}\right| d \xi\right\} \leq \frac{R(x)}{R(a)} \leq \exp \left\{\frac{1}{2} \int_{a}^{x}\left|\frac{A}{r}-\frac{p}{A}\right| d \xi\right\} .
$$

The proof is obvious.

THEOREM 12. If $r(x) p(x)$ is positive and has a continuous derivative, then ${ }^{2}$

${ }^{2}[f(x)]_{+}=\max [f(x), 0],[f(x)]_{-}=-\min [f(x), 0]$. 


$$
\exp \left\{-\frac{1}{2} \int_{a}^{x}\left[\frac{(r p)^{\prime}}{(r p)}\right]_{+} d \xi\right\} \leq \frac{R(x)}{R(a)} \leq \exp \left\{\frac{1}{2} \int_{a}^{x}\left[\frac{(r p)^{\prime}}{(r p)}\right]_{-} d \xi\right\}
$$

For $a(x)=(r(x) p(x))^{1 / 2}$, equation $(1.10)$ becomes

$$
\frac{d(\log R)}{d x}=-\frac{1}{2}\left[\frac{(r(x) p(x))^{\prime}}{r(x) p(x)}\right] \sin ^{2} \Theta
$$

The inequalities of the theorem now follow from the inequalities

$$
-\left[\frac{(r(x) p(x))^{\prime}}{r(x) p(x)}\right]_{+} \leq-\frac{(r(x) p(x))^{\prime}}{r(x) p(x)} \cdot \sin ^{2} \Theta \leq\left[\frac{(r(x) p(x))^{\prime}}{r(x) p(x)}\right]_{-} .
$$

Theorem 12 is a more general statement of a theorem due to Leighton [ 7 , p. 190] in which it is assumed that $r(x) p(x)$ is a positive, monotone function.

THEOREM 13. If $p(x)=-f(x)$, and $r(x) f(x)$ is positive and has a continuous derivative, then

$$
\frac{R(x)}{R(a)} \leq \exp \left\{\int_{a}^{x} \sqrt{\frac{f}{r}} d \xi+\frac{1}{2} \int_{a}^{x}\left[\frac{(r f)^{\prime}}{r f}\right]_{-} d \xi\right\}
$$

and

$$
\frac{R(x)}{R(a)} \geq \exp \left\{-\int_{a}^{x} \sqrt{\frac{f}{r}} d \xi-\frac{1}{2} \int_{a}^{x}\left[\frac{(r f)^{\prime}}{r f}\right]_{+} d \xi\right\}
$$

Setting $a(x)=(r(x) f(x))^{1 / 2}$, we have

$$
\frac{d(\log R)}{d x}=\sqrt{\frac{f(x)}{r(x)}} \sin 2 \Theta-\frac{1}{2} \frac{(r(x) f(x))^{\prime}}{r(x) f(x)} \sin ^{2} \Theta
$$

The proof of the theorem is now obvious.

In the special case, where $(r(x) f(x))^{\prime} \geq 0$, it follows that

$$
R(x) \leq R(a) \exp \left(\int_{a}^{x} \sqrt{\frac{f}{r}} d^{3} \xi\right) .
$$

This inequality is sharp as the example $r(x) \equiv 1$ and $p(x)=-a^{2}$ shows. 
Theorem 11 shows that

$$
\int_{a}^{\infty} \frac{d x}{r(x)}<+\infty, \quad \int_{a}^{\infty}|p(x)| d x<+\infty
$$

are sufficient conditions that all solutions of equation (1) be bounded. The following result covers the case where $p(x) \leq 0$.

THEOREM 14. If $p(x) \leq 0$, in order that all solutions of equation (1) be bounded in $l$, it is necessary that

$$
\int_{a}^{\infty} \frac{d x}{r(x)}<+\infty
$$

and it is sufficient that both

$$
\int_{a}^{\infty} \frac{d x}{r(x)}<+\infty \quad \int_{a}^{\infty} p(x) d x>-\infty
$$

The proof of the sufficiency follows from Theorem 11.

To prove the necessity, consider the particular solution $y(x)$ of equation (1) which satisfies the initial conditions $y(b)=0, y^{\circ}(b)=1$ for some $b \geq a$. According to the Sturm comparison theorem $y(x)>0$ for all $x>b$. It follows that for $x \geq b$ we have

$$
\left(r(x) y^{\prime}(x)\right)^{\prime} \geq 0
$$

hence, integrating both sides of this inequality, we have

$$
y^{\prime}(x) \geq \frac{r(b) y^{\prime}(b)}{r(x)}=\frac{r(b)}{r(x)} \text {. }
$$

Since all solutions of (1) are assumed to be bounded, $y(x)$, in particular, is bounded by $M$. We have, therefore

$$
M \geq y(x) \geq r(b) \int_{b}^{x} \frac{d \xi}{r(\xi)}
$$

for all $x$, and the theorem is proved.

That

$$
\int_{a}^{\infty} \frac{d x}{r(x)}<+\infty
$$


is not sufficient for boundedness is shown by the Euler equation

$$
\left(x^{2} y^{\prime}\right)^{\prime}-\frac{3}{4} y=0
$$

of which $x^{1 / 2}$ is a particular solution.

We obtain the following theorem, particularly applicable to the case where $p(x) \leq 0$, by writing equation $(1)$ in the following form

$$
\left(r(x) y^{\prime}\right)^{\prime}+\left[-\frac{1}{r(x)}+p(x)+\frac{1}{r(x)}\right] y=0
$$

THE OREM 15. If

$$
\int_{a}^{\infty}\left[p(x)+\frac{1}{r(x)}\right] d x<+\infty
$$

then the general solution of equation ( 1 ) is given by

$$
c_{1}\left[\exp \{g(x)\}\left(1+e_{1}(x)\right)\right]+c_{2}\left[\exp \{-g(x)\}\left(1+e_{2}(x)\right)\right]
$$

where $\lim _{x \rightarrow \infty} e_{i}(x)=0 \quad(i=1,2)$.

Transforming equation (2.1) successively by means of the substitutions

$$
y=\exp \{-g(x)\} z \text {, }
$$

and

$$
y=\exp \{g(x)\} w,
$$

we have, respectively,

$$
\left[r(x) \exp \{-2 g(x)\} z^{\prime}\right\rceil^{\prime}+\left[\frac{1}{r(x)}+p(x)\right] \exp \{-2 g(x)\} z=0
$$

and

$$
\left[r(x) \exp \{2 g(x)\} w^{\prime}\right]^{\bullet}+\left[\frac{1}{r(x)}+p(x)\right] \exp \{2 g(x)\} w=0
$$

According to Theorem 3, equation (2.1 $)^{\prime}$ has the particular solution $1+e_{2}(x)$, $\lim _{x \rightarrow \infty} e_{2}(x)=0$, and according to Theorem 4 , equation $(2.1)^{\prime \prime}$ has the 
particular solution $1+e_{1}(x), \lim _{x \rightarrow \infty} e_{1}(x)=0$. The result now follows from the fact that $\left(1+e_{1}(x)\right) \exp \{g(x)\}$ and $\left(1+e_{2}(x)\right) \exp \{-g(x)\}$ are clearly linearly independent solutions of equation (1).

The following result, due to Kneser, [6] (see also, Morse and Leighton [11] and Wintner [13]) is included here in order to complete the results given in this paper. Consider the equation

$$
y^{\prime \prime}-p(x) y=0
$$

where $p(x)$ is a nonnegative, continuous function on the interval $I$.

THEOREM 16. There exists a principal solution $y(x)$ of equation (2.2) such that

$$
\lim _{x \rightarrow \infty} y(x)=m,
$$

where $m$ is finite. All solutions linearly independent of $y(x)$ are unbounded. If

$$
\int_{a}^{\infty} p(x) d x=+\infty
$$

then $m$ vanishes.

This result can be applied to equation (1).

THEOREM 17. If in equation (1) $p(x)$ is nonpositive, then there is a principal solution $y(x)$ such that

$$
\lim _{x \rightarrow \infty} y(x)=m
$$

where $m$ is finite. All solutions linearly independent of $y(x)$ are unbounded provided

$$
\int_{a}^{\infty} \frac{d x}{r(x)}=+\infty
$$

The limit $m$ is zero if

$$
\int_{a}^{\infty} p(x) d x=-\infty
$$

or if 


$$
\int_{a}^{\infty} \frac{d x}{r(x)}<\infty
$$

If

$$
\int_{a}^{\infty} \frac{d x}{r(x)}=+\infty
$$

we transform equation (1) by the change of variable $t=g(x)$. Equation (1) then goes into

$$
\frac{d^{2} y}{d t^{2}}+p(x(t)) r(x(t)) y=0
$$

on the interval $1 \leq t<+\infty$. The proof of the theorem now follows immediately from the preceding theorem and the remark that

$$
\lim _{t \rightarrow \infty} \int_{1}^{t} r(x(\tau)) p(x(\tau)) d \tau=\lim _{x \rightarrow \infty} \int_{a}^{x} p(\xi) d \xi
$$

If

$$
\int_{a}^{\infty} \frac{d x}{r(x)}<+\infty
$$

we proceed as follows. For the substitution $y=h^{1 / 2}(x) z$, equation $(1)$ is transformed into

$$
\left(h(x) r(x) z^{\prime}\right)^{\prime}+\left(-\frac{1}{4 r(x) h(x)}+p(x) h(x)\right) z=0
$$

Introduce the change of variable $t=-\log h(x)$. Equation (2.4) thus becomes

$$
\frac{d^{2} z}{d t^{2}}+\left[-\frac{1}{4}+p(x(t))_{r}(x(t)) e^{-2 t}\right] z=0 .
$$

Theorem 16 is now applicable to show that there is a solution $z(x)$ of equation (2.5) which has a finite limit. The relation between solutions of equation (2.5) and those of equation ( 1 ) concludes the proof of the theorem.

\section{REFERENCES}

1. W. B. Fite, Concerning the zeros of the solutions of certain differential equations, Trans. Amer. Math. Soc. 19 (1918), 341 - 352. 
2. E. Hille, Non-os cillation theorems, Trans. Amer. Math. Soc. 64 (1948), 234-252.

3. E. L. Ince, Researches into the characteristic numbers of the Mathieu equation, Proc. Roy. Soc. Edinburgh, 46 (1925), 20-29.

4. The real zeros of solutions of a linear differential equation with periodic coefficients, Proc. London Math. Soc. 25 (1926), 53-58.

5. A. Kneser, Untersuchungen über die reelen Nullstellen der Integrale linearer Differentialgleichungen, Math. Ann. 42 (1893), 409-435.

6. - Untersuchung und asymptotische Darstellung der Integrale gewisser Differentialgleichungen bei grossen reelen Werthen des Arguments, J. Reine Angew. Math. 116 (1896), $178-212$.

7. W. Leight on, Bounds for the solutions of a second order linear differential equation, Proc. Nat. Acad. Sci. 35 (1949), 190-191.

8. - Principal quadratic functionals and self-adjoint second-order differential equations, Proc. Nat. Acad. Sci. 35 (1949), $192-193$.

9. - On self-adjoint differential equations of second order, Proc. Nat. Acad. Sci. 35 (1949), 656-657.

10. The detection of the oscillation of solutions of a second-order linear differential equation, Duke J. Math. 17 (1950), 57-62.

11. M. Morse, and W. Leighton, Singular quadratic functionals, Trans. Amer. Math. Soc. 40 (1936), $252-286$.

12. A. Wiman, Über die reelen Lösungen der linearen Differentialgleichungen zweiter Ordnung, Ark. Mat. (1917), 22.

13. A. Wintner, On the Laplace-Fourier transcendents occurring in mathematical physics, Amer. J. Math. 69 (1947), 87-98.

14. 117.

WASHINGTON UNIVERSITY, ST. LOUIS, MO. 




\section{Pacific Journal of Mathematics}

\section{Vol. 5, No. $1 \quad$ September, 1955}

Frank Herbert Brownell, III, Flows and noncommuting projections on

Hilbert space ................................... 1

H. E. Chrestenson, A class of generalized Walsh functions ............. 17

Jean Bronfenbrenner Crockett and Herman Chernoff, Gradient methods of maximization ................................... 33

Nathan Jacob Fine, On groups of orthonormal functions. I .......... 51

Nathan Jacob Fine, On groups of orthonormal functions. II ............ 61

Frederick William Gehring, A note on a paper by L. C. Young .......... 67

Joachim Lambek and Leo Moser, On the distribution of Pythagorean

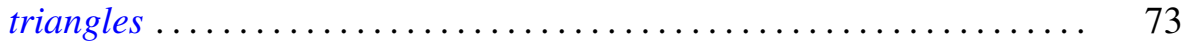

Roy Edwin Wild, On the number of primitive Pythagorean triangles with

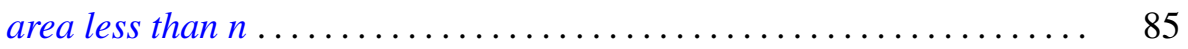

R. Sherman Lehman, Approximation of improper integrals by sums over multiples of irrational numbers ........................ 93

Emma Lehmer, On the number of solutions of $u^{k}+D \equiv w^{2}(\bmod p) \ldots 103$

Robert Delmer Stalley, A modified Schnirelmann density............... 119

Richard Allan Moore, The behavior of solutions of a linear differential equation of second order............................. 125

William M. Whyburn, A nonlinear boundary value problem for second order differential systems. 